\title{
Combination of a SARS-CoV-2 IgG Assay and RT-PCR for Improved COVID-19 Diagnosis
}

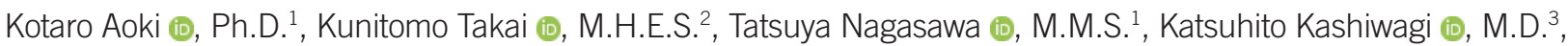

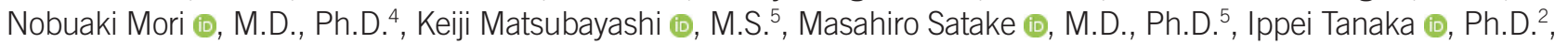

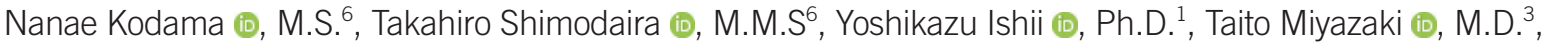

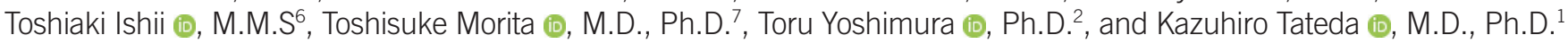

${ }^{1}$ Department of Microbiology and Infectious Diseases, Toho University School of Medicine, Tokyo, Japan; ${ }^{2}$ Research and Development, Abbott Japan LLC, Chiba, Japan; ${ }^{3}$ General Medicine and Emergency Center (Internal Medicine), Toho University Omori Medical Center, Tokyo, Japan; ${ }^{4}$ Department of General Internal Medicine and Infectious Diseases, National Hospital Organization Tokyo Medical Center, Tokyo, Japan; ${ }^{5}$ Central Blood Institute, Blood Service Headquarters, Japanese Red Cross Society, Tokyo, Japan; ${ }^{6}$ Department of Clinical Laboratory, Toho University Omori Medical Center, Tokyo, Japan;

${ }^{7}$ Department of Laboratory Medicine, Toho University School of Medicine, Tokyo, Japan

Background: Coronavirus disease 2019 (COVID-19), caused by severe acute respiratory syndrome coronavirus 2 (SARS-CoV-2), is generally diagnosed by reverse transcription (RT)-PCR or serological assays. The SARS-CoV-2 viral load decreases a few days after symptom onset. Thus, the RT-PCR sensitivity peaks at three days after symptom onset (approximately 80\%). We evaluated the performance of the ARCHITECT ${ }^{\circledR}$ SARS-CoV-2 IgG assay (henceforth termed IgG assay; Abbott Laboratories, Lake County, IL, USA), and the combination of RT-PCR and the IgG assay for COVID-19 diagnosis.

Methods: In this retrospective study, 206 samples from 70 COVID-19 cases at two hospitals in Tokyo that were positive using RT-PCR were used to analyze the diagnostic sensitivity. RT-PCR-negative ( $N=166)$, COVID-19-unrelated $(N=418)$, and Japanese Red Cross Society $(N=100)$ samples were used to evaluate specificity.

Results: Sensitivity increased daily after symptom onset and exceeded $84.4 \%$ after 10 days. Specificity ranged from $98.2 \%$ to $100 \%$ for samples from the three case groups. Seroconversion was confirmed from 9 to 20 days after symptom onset in 18 out of 32 COVID-19 cases with multiple samples and from another case with a positive result in the IgG assay for the first available sample.

Conclusions: The combination of RT-PCR and IgG assay improves the robustness of laboratory diagnostics by compensating for the limitations of each method.

Key Words: COVID-19, SARS-CoV-2 IgG, Immunoassay, ARCHITECT ${ }^{\circledR}$, Reverse transcription (RT)-PCR, Sensitivity, Specificity
Received: September 7, 2020

Revision received: November 11, 2020 Accepted: May 10, 2021

Corresponding author: Kotaro Aoki, Ph.D. Department of Microbiology and Infectious Diseases, Toho University School of Medicine, 5-21-16 Omori, Nishi, Ota-ku, Tokyo 143-8540, Japan

Tel: +81-3-3762-4151

Fax +81-3-5493-5415

E-mail: kotaro.aoki@med.toho-u.ac.jp

\section{INTRODUCTION}

Coronavirus disease 2019 (COVID-19) is a respiratory disease caused by the novel coronavirus severe acute respiratory syndrome coronavirus 2 (SARS-CoV-2) [1]. The incubation period of SARS-CoV-2 ranges from one to 14 days, with the majority of symptoms manifesting in three to five days [2, 3].

COVID-19 is diagnosed by SARS-CoV-2 RNA detection in respiratory samples, especially in nasopharyngeal samples, using reverse transcription (RT)-PCR, and, serologically, by SARS-CoV-2 antibody detection in serum or plasma. The time from exposure is considered to have a strong influence on the sensitivity of RT- 
PCR assays; the viral load peaks three days after symptom onset, with a sensitivity of approximately $80 \%$ at this point [4-6]. Inconsistency of RT-PCR results was an issue for diagnosis in $12.5 \%$ and $21.4 \%$ of cases with COVID-19-like symptoms, showing a negative first and positive second RT-PCR results in two previous studies [7, 8]. Additionally, RT-PCR results performed intermittently often fluctuate between negative and positive [7].

In serological assays for SARS-CoV-2 antibodies, IgG and IgM are detected two to three weeks after symptom onset [5]. As of July 2020, 28 serological assay kits launched by 22 manufacturers have achieved Emergency Use Authorization by the U.S. Food and Drug Administration; among these, 21 are chemiluminescence microparticle immunoassays, including chemiluminescent immunoassays (CLIAs) and ELISAs [9].

The SARS-CoV-2 IgG assay and other serological assays have played important roles in the diagnosis and surveillance of COVID-19, but the clinical utility of such assays remains unclear. We hypothesized that COVID-19 diagnostic sensitivity may be improved by taking advantage of the difference in diagnostic sensitivity peaks between RT-PCR and serological assays with the combination of these methods.

We evaluated the performance of the ARCHITECT ${ }^{\circledR}$ SARSCoV-2 IgG assay (henceforth termed IgG assay; Abbott Laboratories, Lake County, IL, USA) and the combination of RT-PCR and the IgG assay for COVID-19 diagnosis. We assessed results obtained by these methods in confirmed COVID-19 cases at two medical facilities in Tokyo retrospectively and longitudinally. This study can enrich the currently limited data on the IgG assay for Japanese samples.

\section{MATERIALS AND METHODS}

\section{Diagnosis of COVID-19 and clinical information}

This study was approved by the Ethics Committee of Faculty of Medicine, Toho University, Tokyo, Japan (No. A20028_A20020_ A20014_A19099). Blood samples were collected from COVID-19 patients who agreed to participate in this study, on the day of admission, and approximately 3, 7, and 14 days after admission. Samples collected for routine laboratory tests were also analyzed. All serum and plasma samples for the serological assay were stored at $-80^{\circ} \mathrm{C}$ before use. COVID-19 samples were stored for less than three months.

COVID-19 was diagnosed on the basis of SARS-CoV-2 RNA detection in a nasopharyngeal swab using RT-PCR. At Toho University, the laboratory-developed test (LDT) employing SARS-CoV-2 RT-PCR targets the $N$ gene, which encodes the nucleocapsid protein [10]. Total RNA was extracted from nasopharyngeal swabs using QIAamp Viral RNA Mini Kit (Qiagen, Hilden, Germany) or BD MAX ${ }^{\mathrm{TM}}$ EXK TNA-3 (swab; Becton Dickinson, Franklin Lakes, NJ, USA). One-step RT-PCR was performed using QuantStudio $^{\circledR} 5$ (Thermo Fisher Scientific/Applied Biosystems, Waltham, MA, USA) or BD MAX ${ }^{\top M}$ Open System (BD) with TaqMan Fast Virus 1-Step Master Mix (Thermo Fisher Scientific) and BD MAXTNA MMK (SPC) one-step RT-PCR master mix. The primer set (NIID_2019-nCOV_N_F2 and NIID_2019-nCOV_N_ R2) and a FAM-labeled probe (NIID_2019-nCOV_N_P2), which target the $\mathrm{N}$ gene, were used for amplification as previously reported [10]. The limits of detection (LODs) of the two LDT RTPCR methods were evaluated using a $1 \times 10^{5} / \mathrm{mL}$ AccuPlex SARS-CoV-2 Verification Panel (SeraCare, Milford, MA, USA) progressively diluted with RPMI-1640 medium (Thermo Fisher Scientific) containing $1 \%(\mathrm{w} / \mathrm{v})$ fetal bovine serum (Thermo Fisher Scientific). The LODs of the QuantStudio ${ }^{\circledR} 5$ and BD MAXTM assays were 180 viral copies/mL (the verification kit was diluted 550 times) and approximately 330 viral copies $/ \mathrm{mL}$ (the verification kit was diluted 300 times) in the initial sample, respectively. SARS-CoV-2 RT-PCR of samples from the National Hospital Organization Tokyo Medical Center was conducted as an administrative assay at a public research institution in Tokyo. RNA was extracted using QIAamp Viral RNA Mini Kit with a primer set and probe targeting the $N$ and orflab genes as previously reported [11]. The LOD of the SARS-CoV-2 RT-PCR at the public research institution in Tokyo has not been published.

\section{Sensitivity and seroconversion of samples}

In total, 206 samples from 70 cases, including multiple samples available from 32 cases, were collected at Toho University Omori Medical Center and the National Hospital Organization Tokyo Medical Center, Tokyo, Japan, between March and May 2020. These samples were confirmed as positive for SARS-CoV-2 by RT-PCR.

\section{Specificity}

We used three groups of samples for specificity evaluation. The first group comprised 166 samples from 109 cases of suspected COVID-19 judged to have a viral load below the LOD of RT-PCR for SARS-CoV-2. These samples were collected at Toho University Omori Medical Center and the National Hospital Organization Tokyo Medical Center between March and May 2020. The second group comprised 418 samples from 418 cases not suspected of having COVID-19 (collected at Toho University Omori Medical Center between March and June 2020). The third group 
comprised 100 samples collected in 2019 (prior to the COVID-19 outbreak) provided by the Japanese Red Cross (JRC).

\section{Measurement of SARS-CoV-2 lgG}

Samples were analyzed using the IgG assay on an ARCHITECT ${ }^{\circledR}$ i2000SR analyzer (Abbott Laboratories) per the manufacturer's instructions. The IgG assay is a fully automated CLIA for SARSCoV-2 IgG detection in human serum or plasma. Samples, SARSCoV-2 recombinant nucleocapsid antigen ( $\mathrm{N}$ protein)-coated microparticles, and assay diluent were mixed for IgG binding. After washing, anti-human IgG acridinium-labeled conjugate was added to the mixture for binding to the SARS CoV-2 IgG captured on the microparticles. After washing, Pre-Trigger and Trigger Solutions (Abbott Laboratories) were added to start the chemiluminescent reaction, measured in relative light units and converted to the concentration of SARS-CoV-2 IgG in the sample. The cutoff index for positive and negative results was 1.40 .

\section{Statistical analysis}

Sensitivity and specificity were calculated along with the twosided 95\% confidence intervals (Cls) using R software version 3.3.3 (R Foundation for Statistical Computing, Vienna, Austria).
Sensitivity was calculated every three days after symptom onset.

\section{RESULTS}

\section{Sensitivity}

The sensitivity increased at $\leq 3,4-6,7-9,10-12,13-15,16-$ 18 , and $\geq 19$ days after symptom onset (Fig. 1 ). Of the three IgG assay-negative samples collected 16 days after symptom onset, two had index values close to the cutoff. These two samples were collected from the same patient (case 111) on days 18 and 19; a sample collected on day 20 was positive in the IgG assay (index value: 1.52; Fig. 1). The other sample (clearly negative) was collected from a patient with mild symptoms (case M42) 22 days after cough onset.

\section{Specificity}

Assay specificities are shown in Fig. 2. In the group of non-COVID-19 (without an RT-PCR-positive history), the three IgG assay-positive samples were collected from the same patient (case 65). The IgG index in these samples were 3.36 on day $3,3.47$ on day 5 , and 2.97 on day 8 ; the typical increase in the IgG index during the days after symptom onset was not observed, de-

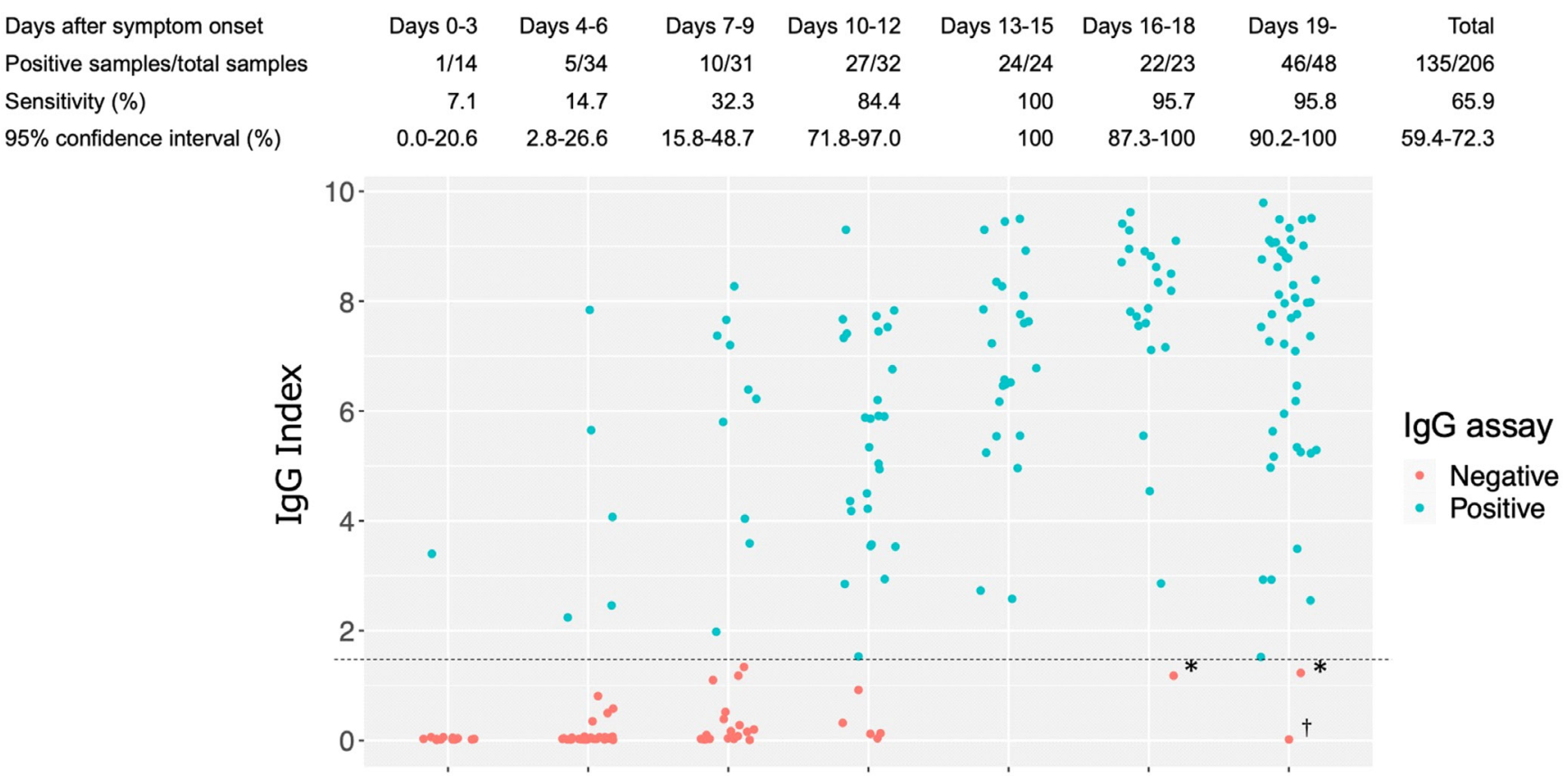

Fig. 1. Sensitivity of the ARCHITECT ${ }^{\circledR}$ SARS-CoV-2 IgG assay for COVID-19 diagnosis. The SARS-CoV-2 IgG index is plotted according to the number of days after symptom onset for 206 samples collected from 70 confirmed COVID-19 cases. * Samples collected from the same patient (case 111) on days 18 and 19 and a sample collected on day 20 were positive in the lgG assay (index value: 1.52). ${ }^{\dagger}$ Sample collected from a patient (case M42) with mild symptoms at 22 days after cough onset and a nasopharyngeal swab obtained at the same time tested positive by the SARS-CoV-2 RT-PCR assay. The dashed line indicates the IgG assay cutoff value (index $=1.40$ ).

Abbreviations: SARS-CoV-2, severe acute respiratory syndrome coronavirus 2; COVID-19, Coronavirus disease 2019; RT-PCR, reverse transcription-PCR. 
spite positivity in the lgG assay (Fig. 2).

\section{Seroconversion of SARS-CoV-2 lgG}

In 18 of the 32 COVID-19 cases with longitudinal observations, seroconversion (i.e., serological assay result change from negative to positive) was observed from day 9 to 20 , and 15 cases were tested positive within 12 days after symptom onset (Fig. 3A). The IgG index value peaked within at least 30 days after symptom onset in nearly all cases. The three cases with delayed seroconversion (case 11, 89, and 111) were not characterized by severity of symptoms (mild, no oxygen administration or ventilator required; moderate, oxygen administration required; and severe, ventilator required). In the 14 cases with the first sample being tested positive in the IgG assay, the IgG index values of five cases (20, 56, 91, 98, and 110) peaked on days 17-24 after symptom onset and then began to decline (Fig. 3B). None of these cases showed negative results in the IgG assay within approximately three months after symptom onset, and the largest decrease in the IgG index (from 9.62 to 7.09 in 43 days) was observed for case 98.

\section{Longitudinal observation of SARS-CoV-2 RT-PCR and IgG assay results}

The RT-PCR and IgG assay results for available samples from
18 cases with seroconversion are shown in Fig. 4A. The duration from symptom onset to RT-PCR positivity ranged from 0 to 15 days (median: 5.5 days) and that from symptom onset to IgG assay positivity ranged from 9 to 52 days (median: 12 days). The interval between the first RT-PCR and IgG assay-positive results from symptom onset ranged from 2 to 51 days (median: 7 days). In 12 out of 18 cases, the RT-PCR result was positive on the same day or later than the day on which the IgG assay result was positive. In case 89, samples were not available for 51 days. Two cases (case 52 and 67) with the RT-PCR result changing from positive to negative within 10 days of symptom onset were positive for the IgG assay on day 10 after symptom onset (Fig. $4 A)$. Nine days after symptom onset, varying RT-PCR results were observed for four cases (11, 13, 88, and 106), but the IgG assay result remained clearly positive (Fig. 4A). The longitudinal observations of RT-PCR and IgG assay results for 14 samples that tested positive in the IgG assay (collected 2 to 16 days after symptom onset) are shown in Fig. 4B. In 11 out of 14 cases, the RT-PCR results were positive on the same day or later than the day on which the IgG assay results were positive. Fluctuating RT-PCR results were observed in six cases (case 3, 50, 56, 91, 107, and 108; Fig. 4B). Of these 32 cases, RT-PCR and lgG assay results of four cases (case 67, 84, 86, and 97) were reversed (RT-PCR turned negative and the lgG assay turned posi-
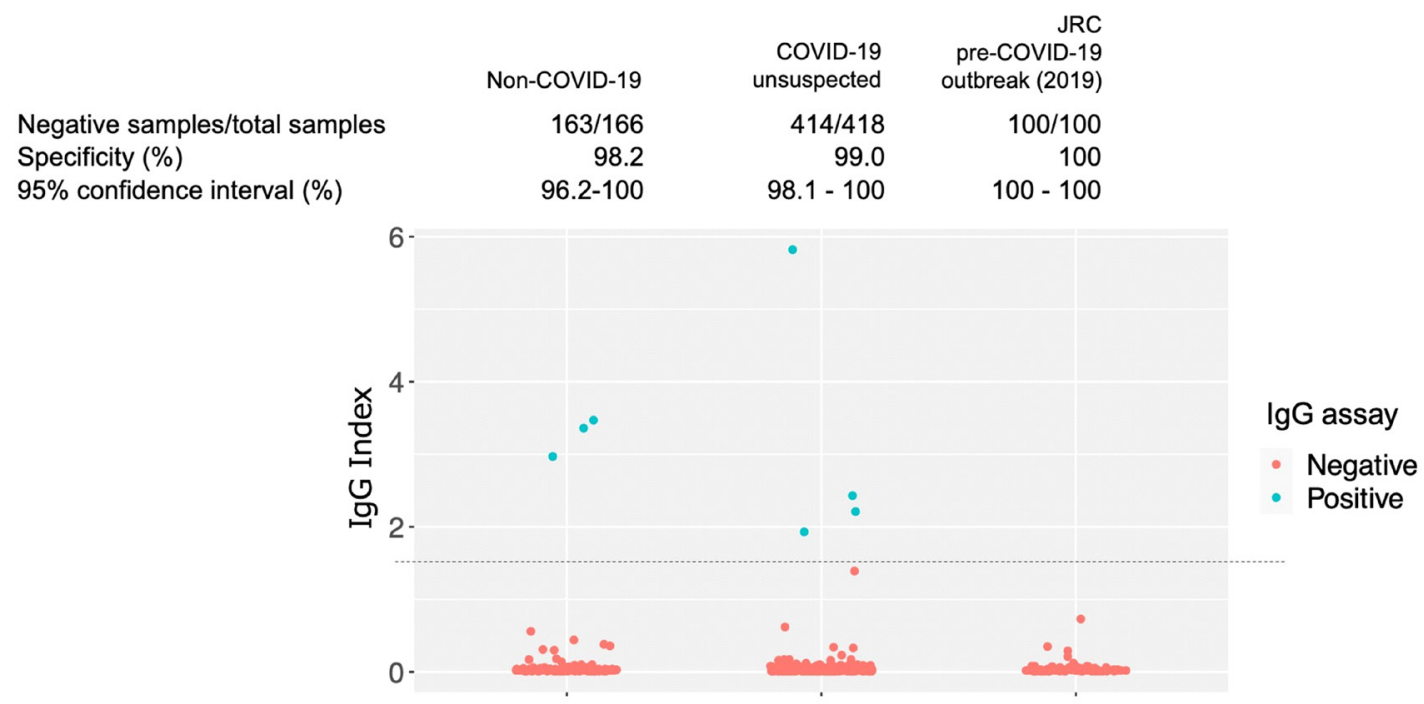

Fig. 2. Specificity of the ARCHITECT ${ }^{\circledR}$ SARS-CoV-2 IgG assay for COVID-19 diagnosis. IgG assay plot of cases and controls, including nonCOVID-19 cases and cases not suspected to be COVID-19. "Non-COVID-19": non-COVID-19 samples collected at Toho University Omori Medical Center and the National Hospital Organization Tokyo Medical Center between March and May 2020; "COVID-19 unsuspected": samples not suspected of COVID-19 collected at Toho University Omori Medical Center; "JRC pre-COVID-19 outbreak": blood donation samples provided by the Japanese Red Cross Society. Three IgG assay-positive samples collected at Toho University Omori Medical Center, which were non-COVID-19, were collected from one patient (case 65). The dashed line indicates the IgG assay cutoff value (index=1.40). Abbreviations: SARS-CoV-2, severe acute respiratory syndrome coronavirus 2; COVID-19, Coronavirus disease 2019; JRC, Japanese Red Cross Society. 

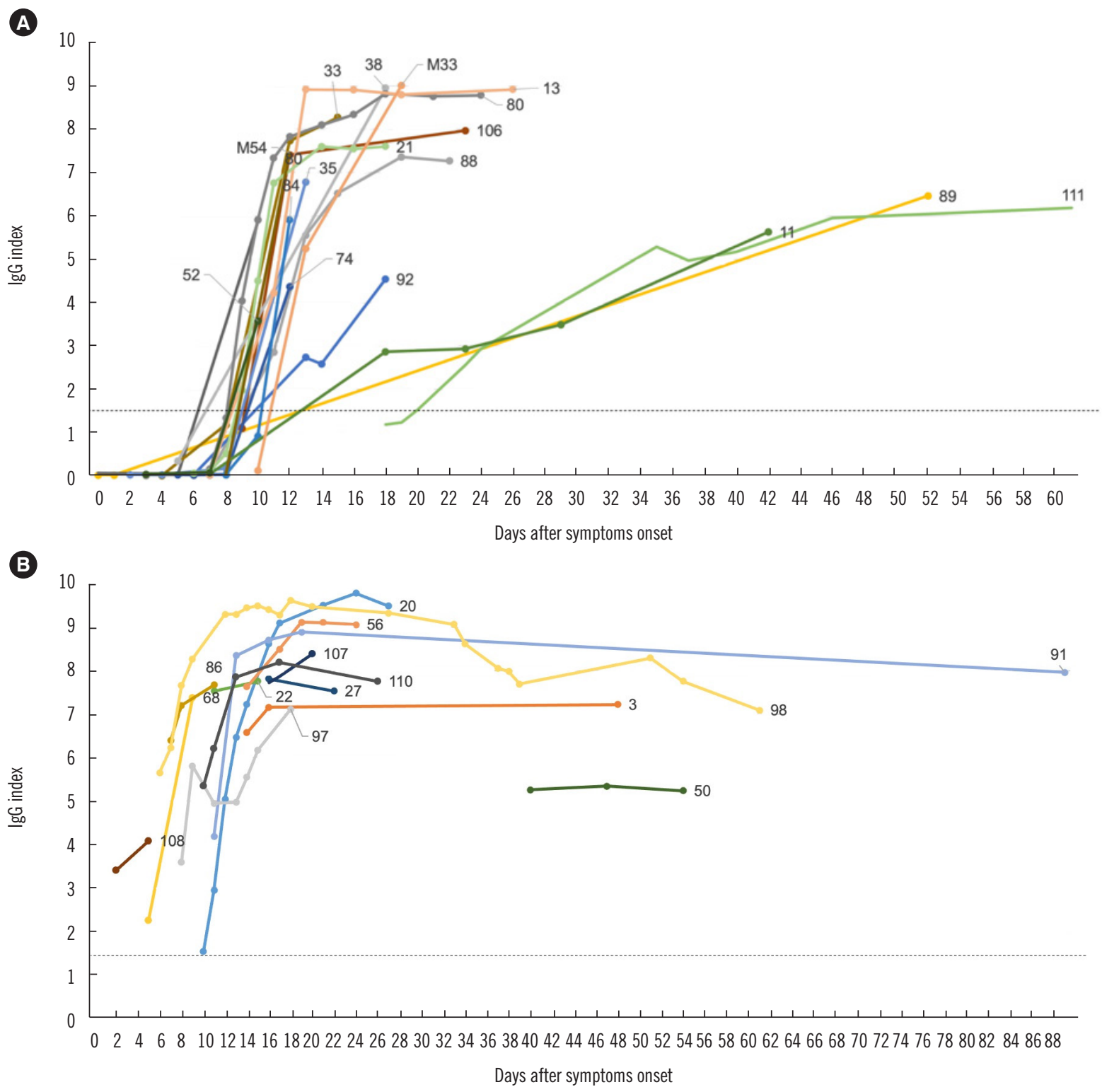

Fig. 3. Longitudinal observation of SARS-CoV-2 IgG assays in COVID-19 cases. (A) Changes in the SARS-CoV-2 IgG index in 18 COVID-19 cases that showed seroconversion (i.e., serological assay result change from negative to positive). (B) Longitudinal observation of SARS-CoV-2 IgG in 14 COVID-19 cases positive for SARS-CoV-2 IgG in the first antibody assay. The dashed line indicates the IgG assay cutoff value (index $=1.40$ ).

Abbreviations: SARS-CoV-2, severe acute respiratory syndrome coronavirus 2; COVID-19, Coronavirus disease 2019.

tive) between 10 and 12 days after symptom onset. Fluctuating RT-PCR results (between negative and positive) were observed in 10 cases (Fig. 5).

\section{DISCUSSION}

We observed longitudinal results of SARS-CoV-2 RT-PCR and IgG assays after symptom onset retrospectively and determined the characteristics of each assay for COVID-19 diagnosis. The
IgG assay showed more reliable longitudinal results than RT-PCR and compensated for the instability of RT-PCR for diagnosing COVID-19 using samples collected at least nine days after symptom onset.

Sensitivity analysis revealed more than $84.4 \%$ agreement between COVID-19 infection and SARS-CoV-2 IgG elevation 10 days after symptom onset; the SARS-CoV-2 IgG positivity increased daily (Fig. 1). Several SARS-CoV-2 serological studies in the USA using the ARCHITECT $^{\circledR}$ platform have reported that $90 \%$ or 


\section{A}

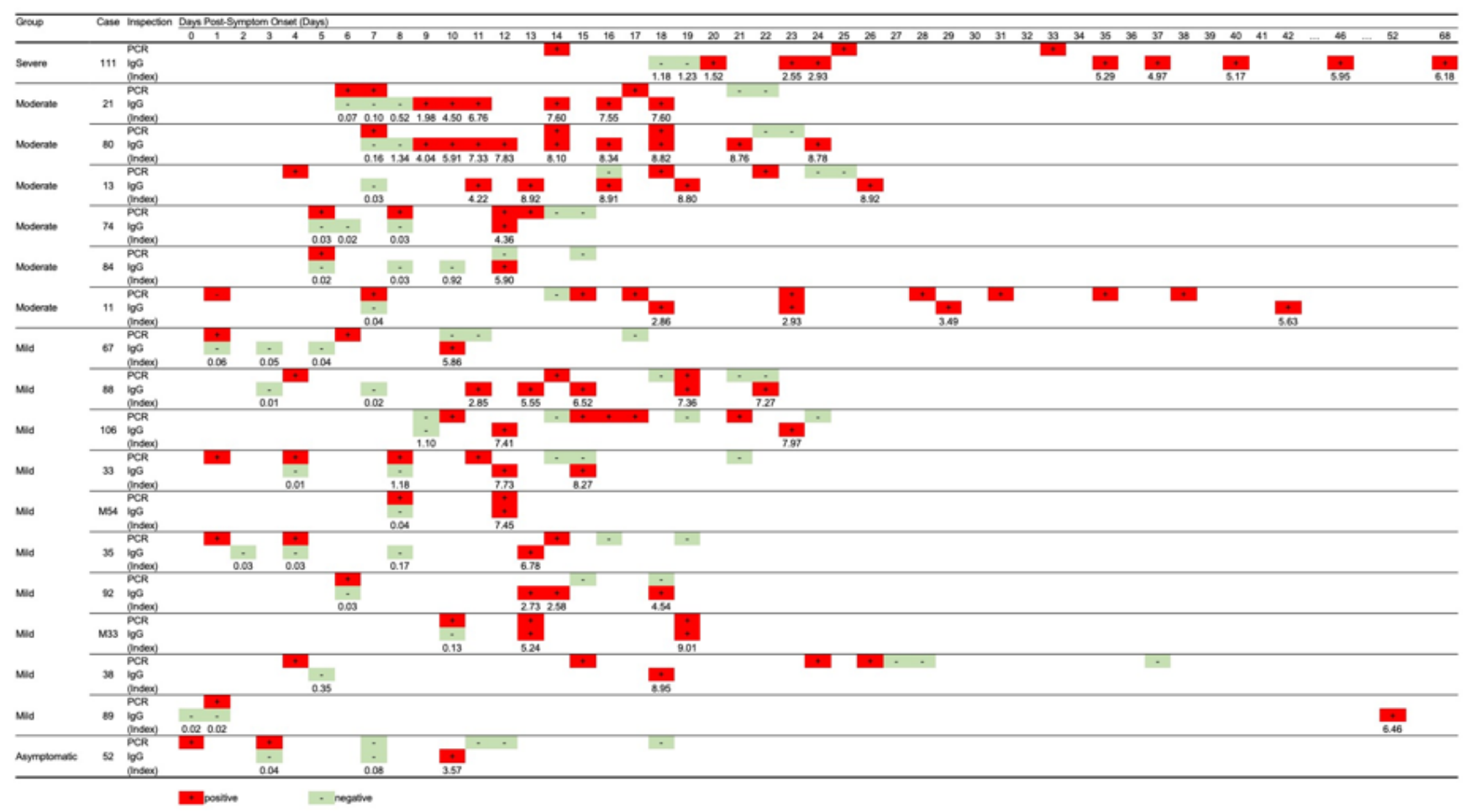

\section{B}

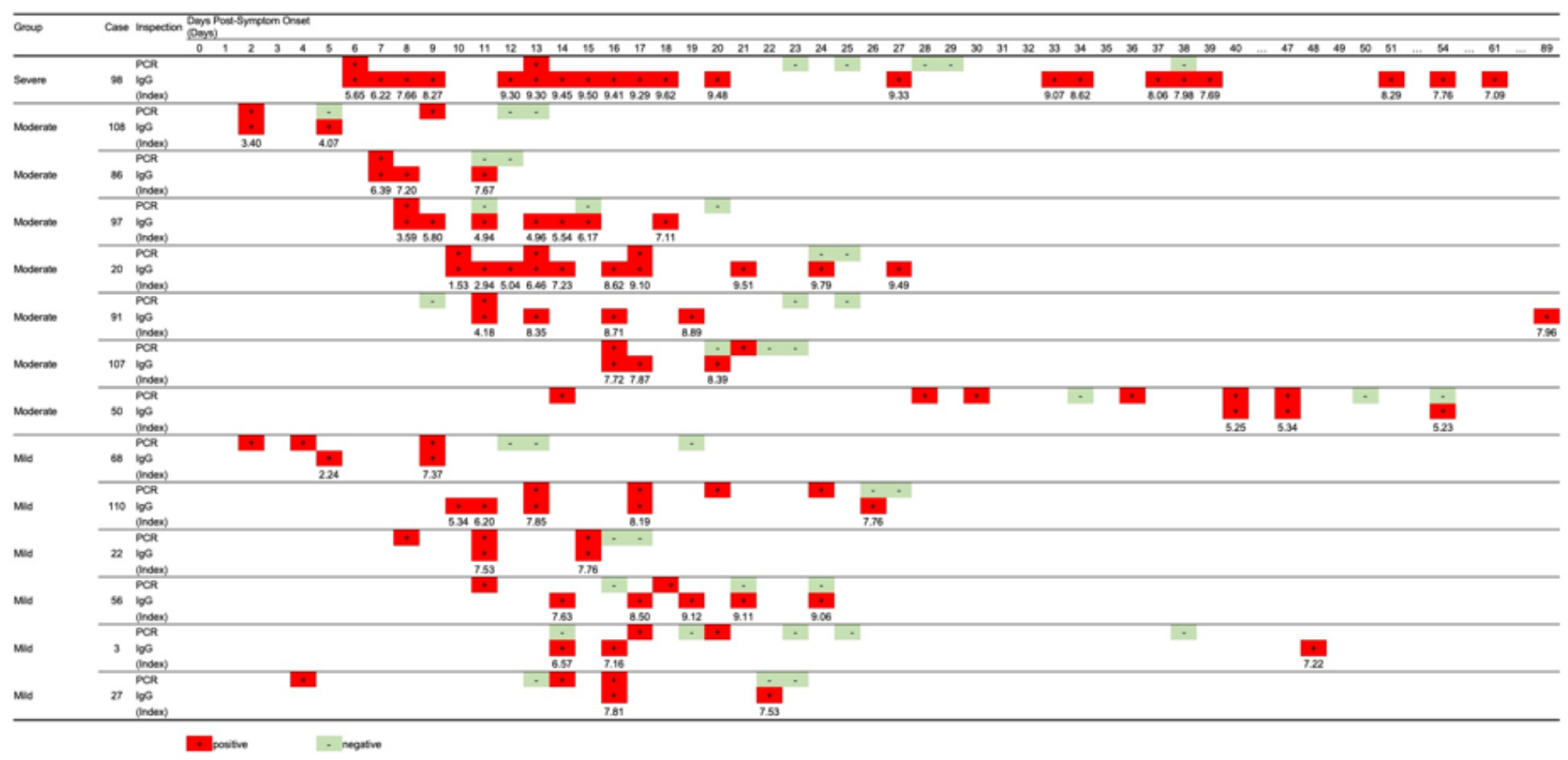

Fig. 4. Longitudinal observation of SARS-CoV-2 RT-PCR and IgG assays in COVID-19 cases. (A) RT-PCR results and the IgG assay index value for the cases shown in Fig. 3A (seroconversion observed). (B) RT-PCR results and the IgG assay index values for the cases shown in Fig. 3B (positive for lgG in the first antibody assay).

Abbreviations: SARS-CoV-2, severe acute respiratory syndrome coronavirus 2; COVID-19, Coronavirus disease 2019; RT-PCR, reverse transcription-polymerase chain reaction. 

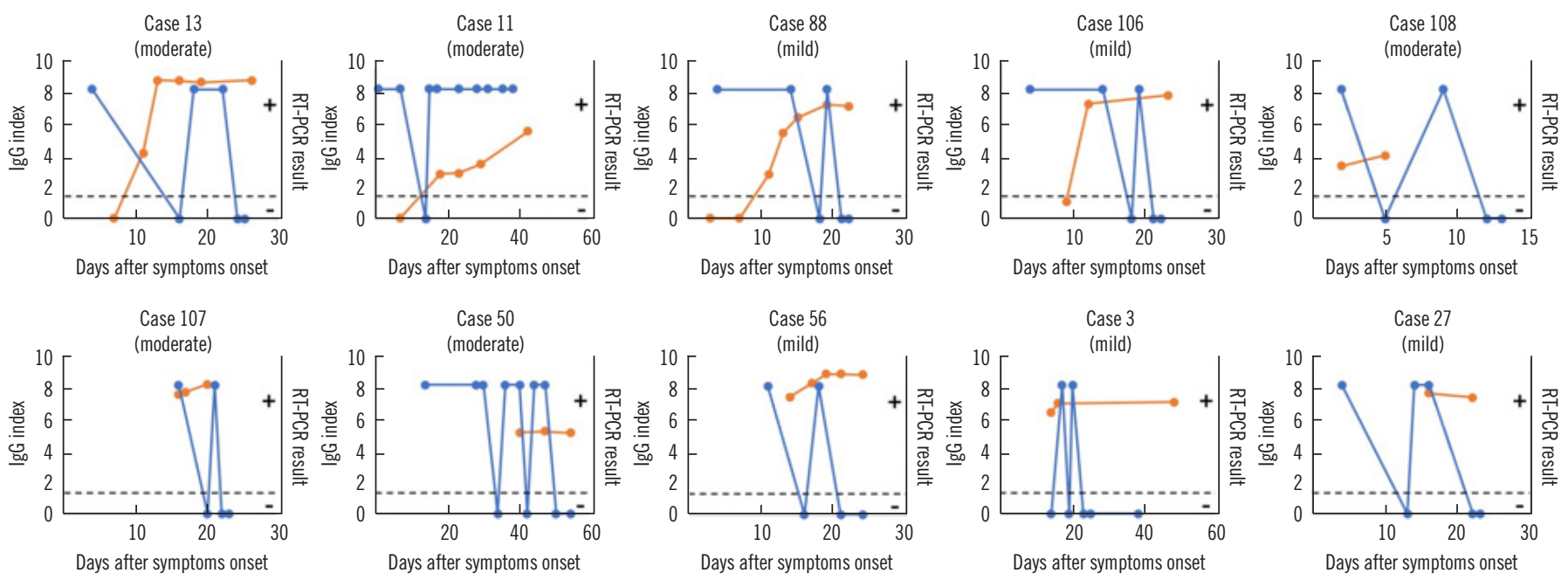

Fig. 5. Longitudinal observation of SARS-CoV-2 RT-PCR and IgG assays in COVID-19 cases with fluctuating RT-PCR results. Orange dots indicate IgG results and the dashed line shows the IgG assay cutoff value (index $=1.40$ ). Blue dots indicate RT-PCR results (positive and negative).

Abbreviations: SARS-CoV-2, severe acute respiratory syndrome coronavirus 2; COVID-19, Coronavirus disease 2019; RT-PCR, reverse transcription-polymerase chain reaction.

more of the samples collected from COVID-19 cases needed to be collected more than 14 days after symptom onset to be positive in the IgG assay [12-14]. In this study, sensitivity was assessed by grouping the days after symptom onset into shorter periods than those in previous studies [12-14]. We found three IgG assay-negative samples even 16 days after symptom onset (samples indicated by "*" and " $\dagger$ " in Fig. 1). Case M42 involved mild COVID-19 symptoms 22 days after cough onset, and a nasopharyngeal swab performed at the same time tested positive by SARS-CoV-2 RT-PCR. Thus, we concluded that the initial cough was unrelated to SARS-CoV-2 infection and the patient had early-stage COVID-19 at the time of sampling. The apparently low IgG values in this sample also point to the possibility of a false-positive RT-PCR result.

The IgG assay specificity was very similar to the range of $99.35 \%-$ 99.90\% reported previously in the USA [12-14] (Fig. 2). The results for the three IgG assay-positive samples were probably false positives, because these samples were collected from the same patient (case 65), who tested negative in SARS-CoV-2 RTPCR and the IgG index did not increase after symptom onset. Excluding this case, the assay specificity in the non-COVID-19 group was $100 \%$. According to the IgG assay instruction manual (https://www.fda.gov/media/137910/download), four samples were suspected to have false-positive results among 1,070 samples assumed to be negative for COVID-19 (997 prior to the COVID-19 outbreak and 73 other respiratory diseases). Our data are similar to the manufacturer's results.
As samples collected at least 10 days after symptom onset showed $84.4 \%$ sensitivity in the IgG assay, serological testing is useful for COVID-19 diagnosis. Assuming that the 32 patients with longitudinal data available for this study came to the hospital between 10 and 12 days after symptom onset, 19 cases could have been diagnosed by RT-PCR (those with positive RT-PCR results 10-12 days after symptom onset). A combination of RTPCR and the IgG assay would facilitate a diagnosis of seven COVID-19 cases (case 52, 67, 68, 84, 86, 97, 108) in addition to those diagnosed using RT-PCR alone. Based on the SARS-CoV-2 viral load kinetics in previous reports $[7,8,15]$ and in this study, RT-PCR results often fluctuate between positive and negative around the LOD. Therefore, the combination of RT-PCR and the IgG assay improved accuracy for COVID-19 diagnosis. RT-PCR compensates for the low sensitivity of the IgG assay, which in turn compensates for the fluctuation in RT-PCR results in the acute COVID-19 phase and the low sensitivity of RT-PCR in the late COVID-19 phase. Indeed, IgG assay positivity might be maintained for at least three months in a limited number of cases. Additionally, as an Italian report showed that $87.4 \%$ of patients recovering from COVID-19 had some persistent symptoms [16], the IgG assay might enable making a diagnosis for a patient with symptoms indicating COVID-19 persistence despite a negative RT-PCR result. .

Not all paired samples were collected at the same time or at predetermined intervals (e.g., hospitalization day $0,3,7$, and 14). Therefore, we plan further evaluations using samples col- 
lected at the same time. We used only one lgG assay, which is a CLIA that uses the N protein to capture IgG in serum/plasma samples. Different results may be obtained using other SARSCoV-2 IgG assays. Despite these limitations, this study provides important knowledge because only serological investigations using immunochromatography (also termed lateral-flow) methods have been reported for COVID-19 cases in Japan to date [1719].

In conclusion, the combination of the SARS-CoV-2 RT-PCR and IgG assays improves the robustness for laboratory diagnosis during the course of COVID-19. In the near future, the IgG assay is expected to become one of the routine assays for COVID-19 diagnosis.

\section{ACKNOWLEDGMENTS}

We are grateful to Mr. Kenjiro Matsushita for help with the IgG assay. We also thank Mitchell Arico at Edanz Group (https://enauthor-services.edanzgroup.com/ac) for editing a draft of this manuscript.

\section{AUTHOR CONTRIBUTIONS}

Aoki K, Takai K, and Tanaka I wrote the manuscript. Ishii Y conceptualized the study, supervised the work, was responsible for project administration, and reviewed the manuscript. Yoshimura T, Ishii T, Ishii Y, and Morita T supervised the study. Nagasawa T, Kodama N, and Shimodaira T performed assays. Kashiwagi K, Mori N, Matsubayashi K, Satake M, and Miyazaki T collected and provided samples. Tateda $\mathrm{K}$ was the principal investigator and acquired the research funding.

\section{CONFLICTS OF INTEREST}

Takai K, Tanaka I, and Yoshimura T are employees of Abbott. The other authors have no conflicts of interest to declare.

\section{RESEARCH FUNDING}

This study was supported by Japan Agency for Medical Research and Development (AMED) under Grant Number JP19fk0108113.

\section{ORCID}

Kotaro Aoki

Kunitomo Takai
Tatsuya Nagasawa

Katsuhito Kashiwagi

Nobuaki Mori

Keiji Matsubayashi

Masahiro Satake

Ippei Tanaka

Nanae Kodama

Takahiro Shimodaira

Yoshikazu Ishii

Taito Miyazaki

Toshiaki Ishii

Toshisuke Morita

Toru Yoshimura

Kazuhiro Tateda https://orcid.org/0000-0002-8070-9102

https://orcid.org/0000-0002-9523-7689

https://orcid.org/0000-0002-1433-0276

https://orcid.org/0000-0002-8361-0167

https://orcid.org/0000-0002-4660-6839

https://orcid.org/0000-0001-9735-3379

https://orcid.org/0000-0003-1396-6720

https://orcid.org/0000-0001-7654-0482

https://orcid.org/0000-0002-1943-4648

https://orcid.org/0000-0001-7239-8677

https://orcid.org/0000-0002-4865-5124

https://orcid.org/0000-0002-5781-4000

https://orcid.org/0000-0002-9178-9777

https://orcid.org/0000-0002-6200-6163

\section{REFERENCES}

1. Zhu N, Zhang D, Wang W, Li X, Yang B, Song J, et al. A novel coronavirus from patients with pneumonia in China, 2019. N Engl J Med 2020; 382:727-33.

2. Lauer SA, Grantz KH, Bi Q, Jones FK, Zheng Q, Meredith HR, et al. The incubation period of coronavirus disease 2019 (COVID-19) from publicly reported confirmed cases: estimation and application. Ann Intern Med 2020;172:577-82.

3. Guan WJ, Ni ZY, Hu Y, Liang WH, Ou CQ, He JX, et al. Clinical characteristics of coronavirus disease 2019 in China. N Engl J Med 2020;382: 1708-20.

4. Wang W, Xu Y, Gao R, Lu R, Han K, Wu G, et al. Detection of SARS-CoV-2 in different types of clinical specimens. JAMA 2020;323:1843-4.

5. Sethuraman N, Jeremiah SS, Ryo A. Interpreting diagnostic tests for SARS-CoV-2. JAMA 2020;323:2249-51.

6. Kucirka LM, Lauer SA, Laeyendecker O, Boon D, Lessler J. Variation in false-negative rate of reverse transcriptase polymerase chain reactionbased SARS-CoV-2 tests by time since exposure. Ann Intern Med 2020; 173:262-7.

7. Li Y, Yao L, Li J, Chen L, Song Y, Cai Z, et al. Stability issues of RT-PCR testing of SARS-CoV-2 for hospitalized patients clinically diagnosed with COVID-19. J Med Virol 2020;92:903-8.

8. Xiao AT, Tong YX, Zhang S. False negative of RT-PCR and prolonged nucleic acid conversion in COVID-19: rather than recurrence. J Med Virol 2020; 92:1755-6.

9. FDA, EUA Authorized Serology Test Performance. https://www.fda.gov/ medical-devices/coronavirus-disease-2019-covid-19-emergency-useauthorizations-medical-devices/eua-authorized-serology-test-performance (Updated on 28th April 2021).

10. Shirato K, Nao N, Katano H, Takayama I, Saito S, Kato F, et al. Development of genetic diagnostic methods for novel coronavirus 2019 (nCoV2019) in Japan. Jpn J Infect Dis 2020;73:304-7.

11. Nagashima M, Kumagai R, Yoshida I, Kawakami M, Nagano M, Asakura $\mathrm{H}$, et al. Characteristics of SARS-CoV-2 isolated from asymptomatic carriers in Tokyo. Jpn J Infect Dis 2020;73:320-2.

12. Bryan A, Pepper G, Wener MH, Fink SL, Morishima C, Chaudhary A, et al. Performance characteristics of the Abbott Architect SARS-CoV-2 IgG assay and seroprevalence in Boise, Idaho. J Clin Microbiol 2020;58: 727-8. 
13. Theel ES, Harring J, Hilgart H, Granger D. Performance characteristics of four high-throughput immunoassays for detection of IgG antibodies against SARS-CoV-2. J Clin Microbiol 2020;58:e01243-20.

14. Tang MS, Hock KG, Logsdon NM, Hayes JE, Gronowski AM, Anderson NW, et al. Clinical performance of two SARS-CoV-2 serologic assays. Clin Chem 2020;66:1055-62.

15. Wölfel R, Corman VM, Guggemos W, Seilmaier M, Zange S, Müller MA, et al. Virological assessment of hospitalized patients with COVID-2019. Nature 2020;581:465-9.

16. Carfi A, Bernabei R, Landi F; Gemelli Against COVID-19 Post-Acute Care Study Group. Persistent symptoms in patients after acute COVID-19. JAMA 2020;324: 603-5.
17. Imai K, Tabata S, Ikeda M, Noguchi S, Kitagawa Y, Matuoka M, et al. Clinical evaluation of an immunochromatographic IgM/lgG antibody assay and chest computed tomography for the diagnosis of COVID-19. J Clin Virol 2020;128:104393.

18. Takita M, Matsumura T, Yamamoto K, Yamashita E, Hosoda K, Hamaki T, et al. Geographical profiles of COVID-19 outbreak in Tokyo: an analysis of the primary care clinic-based point-of-care antibody testing. J Prim Care Community Health 2020;11:2150132720942695.

19. Lisboa Bastos M, Tavaziva G, Abidi SK, Campbell JR, Haraoui LP, Johnston JC, et al. Diagnostic accuracy of serological tests for covid-19: systematic review and meta-analysis. BMJ 2020;370:m2516. 